\title{
Reconstrucción de defectos óseos segmentarios postraumáticos mediante técnica de inducción de membrana
}

\begin{abstract}
Surgical treatment of post-traumatic segmental bone defects through the induction membrane technique
\end{abstract}
Julio C. Vejarano-Solano, ${ }^{1, a ; 2, b}$, Carlos F. Ruiz-Semba ${ }^{1, a ; 2, b}$, Carlos J. Ganoza-Arróspide ${ }^{1, a ; 2, b}$, Jorge E. Hurtado-Fernández ${ }^{1, a ; 2, b}$

\section{RESUMEN}

Objetivos: Describir y evaluar los resultados del tratamiento quirúrgico de defectos óseos postraumáticos (DOPT) segmentarios mediante técnica de inducción de membrana. Material y métodos: Estudio descriptivo longitudinal de 20 casos de DOPT segmentarios tratados en el Hospital Nacional Cayetano Heredia de Lima, desde enero de 2009 a junio de 2014, mediante reconstrucción en dos etapas con técnica de inducción de membrana. En la primera etapa se indujo la formación de membrana con un espaciador de cemento óseo impregnado con antibiótico. En la segunda etapa se reemplazó el espaciador por injerto óseo. Se evaluó el tiempo de consolidación, grado de acortamiento de la extremidad y resultado funcional. Se registraron las complicaciones. Resultados: Se incluyeron 20 pacientes (15 varones y 5 mujeres), con edad promedio de 29 años (rango: 13-54). Once casos fueron DOPT en tibia, 7 casos en fémur, uno en radio y uno en cúbito. El 90\% de los DOPT fueron en longitud mayor o igual a $50 \mathrm{~mm}$, con un promedio de $73 \mathrm{~mm} ; 60 \%$ de los casos tenían cultivos positivos al inicio del tratamiento. Presentaron consolidación el $90 \%$ de los casos con un único aporte de injerto óseo, el tiempo promedio para la consolidación fue 8 meses. Uno requirió un segundo aporte de injerto y un caso presentó persistencia de infección sin consolidación. Conclusiones: La técnica de inducción de membrana es un método eficaz para el tratamiento de DOPT segmentarios.

PALABRAS CLAVE: Trasplante óseo, osteomielitis, osteonecrosis, alargamiento óseo. (Fuente: DeCS BIREME).

\section{SUMMARY}

Objective: To describe the results of surgical treatment of post-traumatic segmental bone defects (PSBD) through the induction membrane technique. Methods: Observational study in patients attended at Hospital Nacional Cayetano Heredia in Lima from January 2009 to June 2014, in whom a two-step reconstruction procedure for PSBD was performed using the induction membrane technique. In the first step, the formation of a membrane was induced with an antibiotic spacer block followed by replacement of the spacer with bone grafting. Time to bone consolidation, degree of shortening of the extremity, functional status and complications were recorded. Results: Twenty patients were included in the study (15 males and 5 females); mean age was 29 year (range: 13-54). Eleven cases had PSBD in the tibia, 7 in the femur, 1 in the radius and 1 in the ulna; in 90\% of cases the PSBD had a length of at least 50mm (mean of $73 \mathrm{~mm}$ ); $60 \%$ of cases had a positive culture at the beginning of treatment. Ninety percent consolidated the fracture with a single procedure in a meantime of 8 months. One patient required a second procedure and

\footnotetext{
Centro de Traumatología y Ortopedia, Hospital Nacional Cayetano Heredia. Lima, Perú.

Departamento de Cirugía, Facultad de Medicina Alberto Hurtado, Universidad Peruana Cayetano Heredia. Lima, Perú.

Médico Asistente.

Profesor
} 
one presented a persistent infection without consolidation. Conclusions: The induction membrane technique is an efficacious procedure for managing PSBD.

KEYWORDS: Bone transplant, osteomyelitis, osteonecrosis, bone lengthening. (Source: MeSH NLM)

\section{INTRODUCCIÓN}

Los defectos óseos postraumáticos (DOPT) se producen por traumas de alta energía que ocasionan pérdida aguda de hueso; ocurren en forma subaguda durante el desbridamiento quirúrgico de una fractura expuesta conminuta, y también pueden verse en casos crónicos como seudoartrosis atrófica u osteomielitis difusa con grandes secuestros (1-3). Una situación clínica frecuente son aquellos DOPT presentes en seudoartrosis u osteosíntesis infectadas de fémur y tibia $(4,5)$.

Los DOPT alteran drásticamente la calidad de vida del paciente, y pueden producir serias secuelas como acortamiento disfuncional de la extremidad, deformidad angular, rigidez articular y trastorno irreversible de la marcha. Los DOPT ponen a la extremidad en riesgo de amputación $(1,5,6)$.

Los DOPT se clasifican en tres tipos: Tipo 1, cuando la pérdida ósea afecta a menos del $50 \%$ del diámetro del hueso; tipo 2, si compromete más del $50 \%$, pero se mantiene contacto en uno o más puntos de la cortical, y tipo 3, llamados DOPT segmentarios, cuando el defecto no posee continuidad en ningún punto de la cortical (7).

La reconstrucción de un DOPT segmentario no debe causar mayor morbilidad al paciente. Al término del tratamiento se debe lograr la consolidación manteniendo la longitud y alineación del hueso; erradicar la infección; y conseguir un rango articular funcional (8-10). Clásicamente se han descrito dos opciones principales para reconstruir un DOPT segmentario: peroné vascularizado (11-14) y osteogénesis por distracción (15). Recientemente ha cobrado interés la técnica descrita por Masquelet, que consiste en crear un tejido biológicamente activo, denominado membrana inducida, colocando temporalmente un espaciador de polimetilmetacrilato (PMMA) en el defecto óseo. Esta técnica constituiría una opción eficaz para reconstruir un DOPT segmentario con mínimas complicaciones $(16,17)$.

La técnica de Masquelet o de inducción membrana consta de dos etapas, durante la primera se coloca en el DOPT un espaciador hecho a mano con PMMA impregnado con antibiótico (18-20). El espaciador induce la formación de la membrana de Masquelet, que es un tejido vascularizado rico en factores de crecimiento como el factor de crecimiento del endotelio vascular (VEGF) y factor de crecimiento transformante beta (TGF-ß1) $(21,22)$. La segunda etapa se realiza entre cuatro a ocho semanas después: se retira el espaciador preservando cuidadosamente la membrana inducida y se sustituye por injerto óseo esponjoso autólogo, La función de la membrana inducida es rodear al injerto óseo y nutrirlo, evitando su reabsorción. La estabilidad definitiva del hueso puede realizarse con fijación externa o interna $(19,20)$.

En nuestro medio no existen estudios publicados sobre la técnica de inducción de membrana, por lo que nuestro estudio tuvo como objetivo describir los resultados de su aplicación en la reconstrucción de defectos óseos postraumáticos segmentarios.

\section{MATERIAL Y MÉTODOS}

Estudio descriptivo, longitudinal, tipo serie de casos se realizó en el Centro de traumatología y ortopedia del Hospital Nacional Cayetano Heredia en pacientes con diagnóstico de DOPT segmentario desde enero de 2009 a junio del 2014.

El criterio de inclusión fue todo paciente con DOPT segmentario en el esqueleto apendicular: húmero, cubito, radio, fémur y tibia, que fueron tratados quirúrgicamente usando técnica de inducción de membrana. Se excluyeron a los pacientes con historia clínica o radiografías incompletas y los que abandonaron el tratamiento.

Se tomaron radiografías preoperatorias en proyecciones antero posterior y lateral del hueso comprometido. En casos seleccionados se complementó con tomografía axial computarizada para valorar la extensión de los secuestros y la vitalidad de los segmentos óseos. Se midió la mayor longitud del DOPT segmentario tanto en proyección anterior como lateral, la longitud final considerada para este estudio resultó de promediar ambas longitudes. La evaluación vascular se realizó mediante examen clínico, ecografía 
Doppler o angioresonancia. Se tomó muestras para cultivo y antibiograma.

\section{Técnica quirúrgica de inducción de membrana} (Figura 1)

\section{Primera etapa}

Desbridamiento: Se eliminaron los fragmentos óseos desvitalizados, secuestros, implantes infectados o inestables, partes blandas desvitalizadas o necróticas, fistulas y fibrosis. Se comprobó la vitalidad del hueso con el signo de paprika (5).

Preparación del espaciador: Se utilizó el cemento óseo aplicado rutinariamente en artroplastia cementada, el cual posee dos componentes: $40 \mathrm{~g}$ de polvo de polímero, constituido principalmente por polimetilmetacrilato (PMMA) y 20 mililitros de monómero líquido, constituido principalmente por metilmetacrilato (MMA) (23-25). Se incorporó al PMMA $4 \mathrm{~g}$ de uno o más antibióticos termoestables de presentación en polvo (clindamicina, vancomicina, meropenem y ceftazidima) elegidos tomando en cuenta los siguientes criterios: 1) resultado de cultivo y antibiograma, 2) en osteomielitis crónica con cultivo negativo se eligió vancomicina para cubrir $S$. aureus, 3 ) en pérdida ósea aguda por fractura expuesta sin evidencia de infección se eligió clindamicina, 4) cuando se aisló más de un germen se combinó dos antibióticos. El PMMA y el antibiótico se mezclaron manualmente hasta uniformizarse, acto seguido se agregó el MMA, formándose una pasta. Se esperó unos minutos para alcanzar la fase de trabajo del cemento óseo.

Colocación del espaciador: Se realizó antes del inicio de la fase de endurecimiento a fin de evitar la intrusión excesiva del cemento en el hueso. De forma práctica se utilizó a manera de molde jeringas de plástico que semejaban el diámetro del hueso comprometido. Se esperó que el cemento endurezca y se enfrió con solución salina para disipar el calor de la polimerización.

Manejo de partes blandas: Se aseguró la cobertura del hueso y el espaciador mediante cierre primario cuidadoso o con colgajos musculares o fasciocutáneos.

Estabilización temporal: La estabilización temporal se realizó principalmente con fijadores externos, con

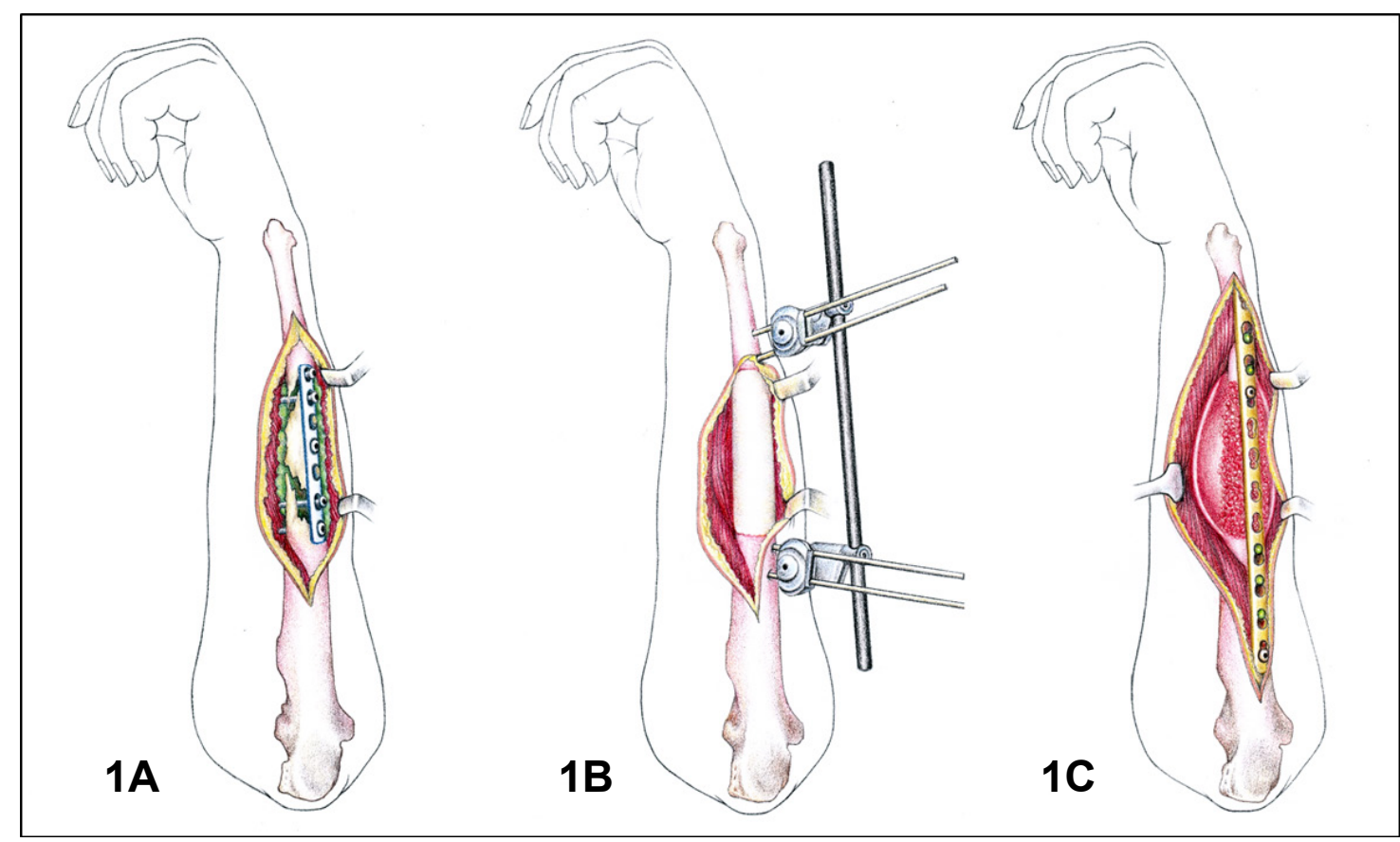

Figura 1. Esquema ilustrativo de defecto óseo en cubito (caso 1) tratado mediante técnica de inducción de membrana. Primera etapa: 1A. Desbridamiento cuidadoso y extirpación de los implantes y tejido óseo infectados (1). 1B. Colocación de espaciador de polimetilmetacrilato (PMMA) impregnado con antibiótico (2) en el defecto óseo y fijación externa temporal (el espaciador inducirá la membrana de Masquelet). Segunda etapa: 1C. reemplazo del espaciador por injerto óseo esponjoso (3) y fijación interna definitiva. La membrana inducida (4) rodea íntegramente al injerto y debe ser suturada cuidadosamente. (Dibujo: Petra Alfaro Burgos) 
tutores internos como clavos rush o steinman insertos dentro del espaciador y férulas de yeso o braces ortopédicos.

\section{Segunda etapa}

Retiro del espaciador: Cuatro a ocho semanas después se procedió al retiro del espaciador cuidando de no dañar la membrana inducida. A la inspección, la membrana inducida debía mostrar las siguientes características: grosor entre 1 a $3 \mathrm{~mm}$; superficie interna no adherida al espaciador, de aspecto epitelial y color rojizo pálido, y superficie externa adherida al plano superior de tejido (19).

Estabilización definitiva: La estabilización definitiva se realizó con fijación externa o interna.

Aporte de injerto: Se utilizó injerto óseo esponjoso tomando como fuente principal la cresta iliaca anterior. En los casos con grandes defectos se complementó con injerto heterólogo o matriz ósea desmineralizada.

Se realizó controles radiográficos seriados a todos los pacientes, documentándose el tiempo en que se observó consolidación y el tiempo en que se permitió carga total. Se registraron los rangos funcionales y las complicaciones.

Para el manejo de los datos se utilizaron tablas diseñadas con el programa Microsoft Excel 2007. Se registraron datos de edad, etiología del defecto óseo, tratamiento previo y resultados de cultivos; antibióticos usados en la preparación del espaciador y su tiempo de permanencia durante la primera etapa; tipo de injerto e implantes utilizados. Se empleó estadística descriptiva con medidas de tendencia central como la media aritmética para el análisis de datos. Los cálculos estadísticos fueron realizados con el programa SPSS versión 16.0.2

El estudio fue revisado y aprobado por el Comité de Ética del Hospital Nacional Cayetano Heredia.

\section{RESULTADOS}

Se incluyeron 20 pacientes con DOPT segmentarios (Tipo 3). En 11 casos estuvo afectada la tibia, en siete el fémur, en uno el cúbito y en uno el radio. La edad promedio de los pacientes fue 29 años (rango: 13 - 54), 15 fueron varones. El $60 \%$ de los pacientes tuvieron cultivos positivos al inicio del tratamiento, siendo los gérmenes aislados: Staphylococcus aureus en 8 pacientes, $P$. aeruginosa en 3, E. coli en 2 pacientes, en 1 caso se aisló más de un germen.

La longitud promedio de los DOPT segmentarios tratados fue de $73 \mathrm{~mm}$ (Tabla 1). Con respecto a la etiología de los DOPT segmentarios, el $45 \%$ correspondían a casos de seudoartrosis séptica con varias cirugías previas y tiempos de enfermedad mayores a 9 meses; el 25\% fueron catalogadas como osteosíntesis infectadas; $15 \%$ correspondían a osteomielitis crónica difusa sin antecedente de osteosíntesis y el 15\% restante eran fracturas expuestas con pérdida aguda de hueso.

Las características de la primera y segunda etapa se detallan en la tabla 2. Para la preparación del espaciador se utilizó vancomicina como único antibiótico en 12 casos, vancomicina en combinación con meropenem o ceftazidima en cuatro, sólo meropenem en un caso y clindamicina en cuatro casos. El tiempo promedio de permanencia del espaciador en el DOPT fue de 8,5 semanas.

Fue necesario realizar colgajos en ocho de los casos tratados en tibia ( 6 colgajos rotacionales y 3 colgajos libres).

Durante la segunda etapa se utilizó fijación interna en el $80 \%$ de los casos. Se usó injerto aútologo en 19 (95\%) pacientes, siendo la fuente de injerto la cresta iliaca en 17 casos y en 2 casos se usó injerto autólogo obtenido mediante sistema de fresado y aspirado del canal femoral (Sistema RIA) (26). En tres casos se complementó con injerto heterólogo. En una paciente de 13 años (caso 2) se usó sólo injerto heterólogo.

El $90 \%$ de los casos consolidaron con un único aporte de injerto óseo. El tiempo promedio para la consolidación fue de 8 meses (5,6-13 meses). El tiempo de seguimiento promedio fue de 2,5 años ( 9 meses-5,5 años).

Un caso con defecto diafisiario infectado con E. coli (caso 6) persistió con infección luego de la segunda etapa, fue necesario el retiro del injerto óseo y colocación de perlas de antibiótico e iniciar un nuevo proceso reconstructivo. Un caso con defecto femoral (caso 8) requirió un segundo aporte de injerto óseo, consolidó en 17 meses y con $3 \mathrm{~cm}$ de acortamiento. El caso 12 con DOPT diafometafisiario proximal de la tibia presentó ruptura de una placa bloqueada de tibia proximal, luego de realizar carga no indicada. Este caso fue solucionado con retiro del implante y 
Tabla 1. Características clínicas de pacientes con defectos óseos postraumáticos segmentarios.

\begin{tabular}{|c|c|c|c|c|c|c|c|}
\hline Caso & Edad & Sexo & Hueso & Localización & $\begin{array}{c}\text { Longitud } \\
(\mathrm{mm})\end{array}$ & Germen aislado & Etiología \\
\hline 1 & 25 & M & cúbito & diafisiario & 70 & S. aureus & osteosíntesis infectada \\
\hline 2 & 13 & $\mathrm{~F}$ & radio & diafisiario & 125 & S. aureus & osteomielitis crónica \\
\hline 3 & 25 & M & fémur & metafisiario proximal & 50 & negativo & osteosíntesis infectada \\
\hline 4 & 36 & $\mathrm{~F}$ & fémur & diafisiario & 75 & negativo & seudoartrosis séptica \\
\hline 5 & 26 & M & fémur & diafisiario & 80 & negativo & osteomielitis crónica \\
\hline 6 & 30 & M & fémur & diafisiario & 85 & E. coli & osteosíntesis infectada \\
\hline 7 & 26 & M & fémur & diafisiario & 55 & P. aeruginosa & seudoartrosis séptica \\
\hline 8 & 54 & $\mathrm{~F}$ & fémur & diafometafisiario distal & 105 & S. aureus & seudoartrosis séptica \\
\hline 9 & 37 & M & fémur & diafometafisiario distal & 80 & negativo & fractura expuesta $\mathrm{III}^{\mathrm{o}}$ \\
\hline 10 & 14 & M & tibia & metafisiario proximal & 45 & S. aureus & osteomielitis crónica \\
\hline 11 & 43 & M & tibia & diafometafisiario proximal & 60 & negativo & fractura expuesta III $^{\circ}$ \\
\hline 12 & 17 & $\mathrm{~F}$ & tibia & diafometafisiario proximal & 65 & negativo & fractura expuesta $\mathrm{III}^{\circ}$ \\
\hline 13 & 41 & M & tibia & diafisiario & 95 & S. aureus + Pseudomona. & seudoartrosis séptica \\
\hline 14 & 28 & M & tibia & diafisiario & 80 & S. aureus & seudoartrosis séptica \\
\hline 15 & 23 & M & tibia & diafisiario & 45 & E. coli & seudoartrosis séptica \\
\hline 16 & 24 & M & tibia & diafisiario & 85 & S. aureus & ostesíntesis infectada \\
\hline 17 & 35 & M & tibia & diafisiario & 70 & P. aeruginosa & seudoartrosis séptica \\
\hline 18 & 43 & $\mathrm{~F}$ & tibia & diafisiario & 60 & negativo & seudoartrosis séptica \\
\hline 19 & 20 & M & tibia & diafometafisiario distal & 75 & S. aureus & osteosíntesis infectada \\
\hline 20 & 25 & M & tibia & diafometafisiario distal & 50 & negativo & seudoartrosis séptica \\
\hline
\end{tabular}

colocación de fijación externa, no siendo necesario un nuevo aporte de injerto. Durante el seguimiento, en un DOPT diafometafisiario distal de tibia (caso 20) que consolidó fue necesario realizar un curetaje de un secuestro residual.

\section{DISCUSIÓN}

Reconstruir un DOPT constituye un reto para el cirujano ortopédico. El aporte directo de injerto óseo es la forma más sencilla de resolver un defecto óseo, pero está restringido a defectos menores a $5 \mathrm{~cm}$ y libres de infección $(1,2)$. En 1989, Cristian y col (27), utilizaron injerto óseo autólogo para tratar defectos óseos de hasta $14 \mathrm{~cm}$ en fracturas expuestas de tibia. Mediante un protocolo en dos etapas: primero rellenaron el defecto óseo con perlas de PMMA impregnadas con cefazolina y tobramicina y 8 semanas después las remplazaron por injerto óseo esponjoso, logrando la consolidación en todos los casos. Este estudio preliminar ponderaba dos propiedades básicas de las perlas y espaciadores de PMMA: liberar antibiótico sin toxicidad sistémica y preservar el espacio libre de fibrosis para una disección más segura $(23,27,28)$.

Masquelet $(16,17)$ perfeccionó el concepto de reconstrucción por etapas de Cristian; reveló la importancia del tejido reactivo que prolifera alrededor del espaciador de PMMA implantado. Este tejido denominado membrana inducida está ricamente vascularizada, y conformada por colágeno Tipo I y células fibroblásticas externamente e internamente semejante a un epitelio sinovial; la membrana secreta factores de crecimiento en alta concentración $(21,22)$. Esta membrana mejora la incorporación del injerto óseo. Masquelet (17), ha tratado con éxito defectos óseos de hasta $24 \mathrm{~cm}$.

Progresivamente ha crecido el interés por la reconstrucción ósea usando espaciadores de PMMA. Representa una alternativa a las técnicas tradicionales de reconstrucción: osteogénesis por distracción e injerto de peroné vascularizado $(9,10)$. 


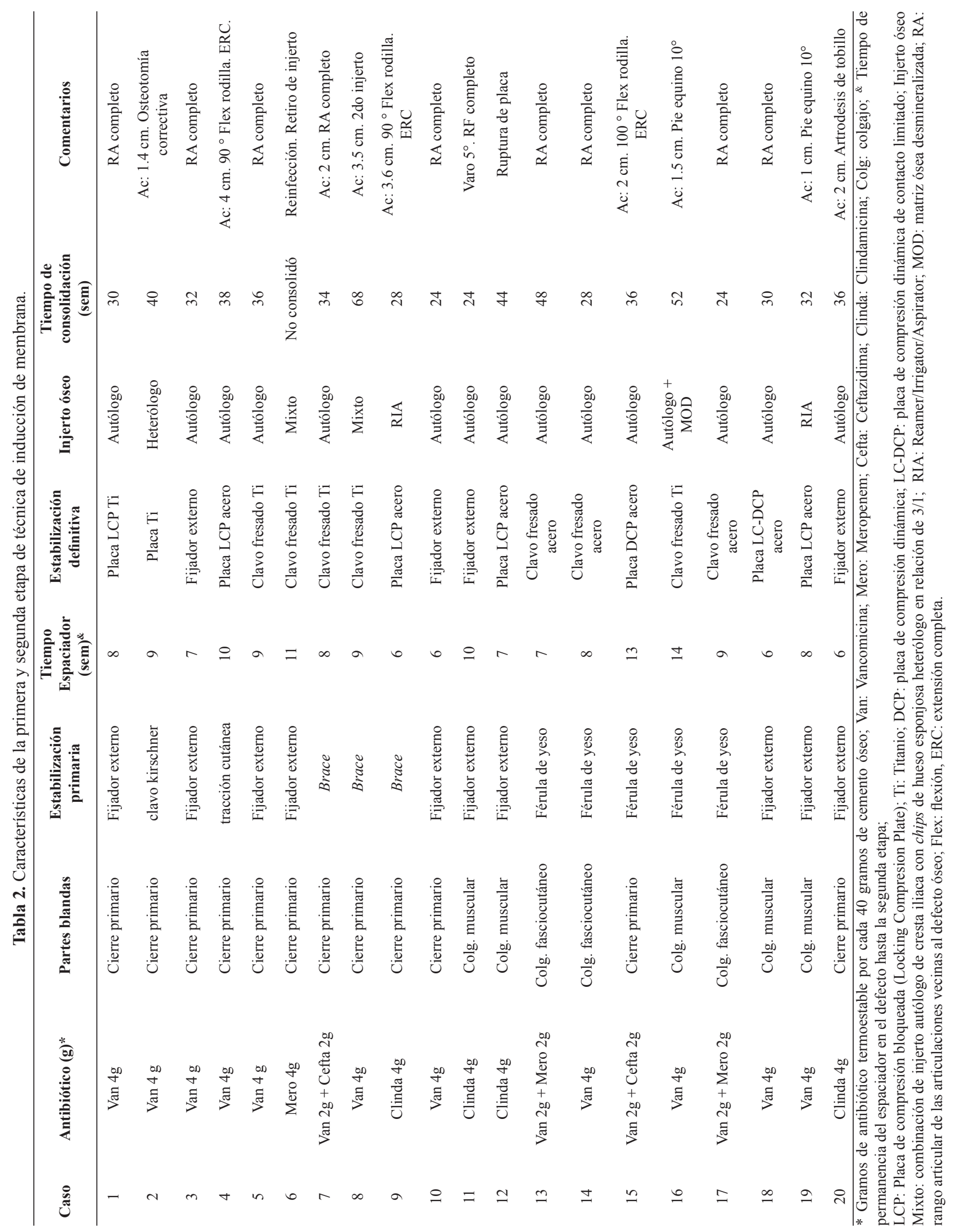



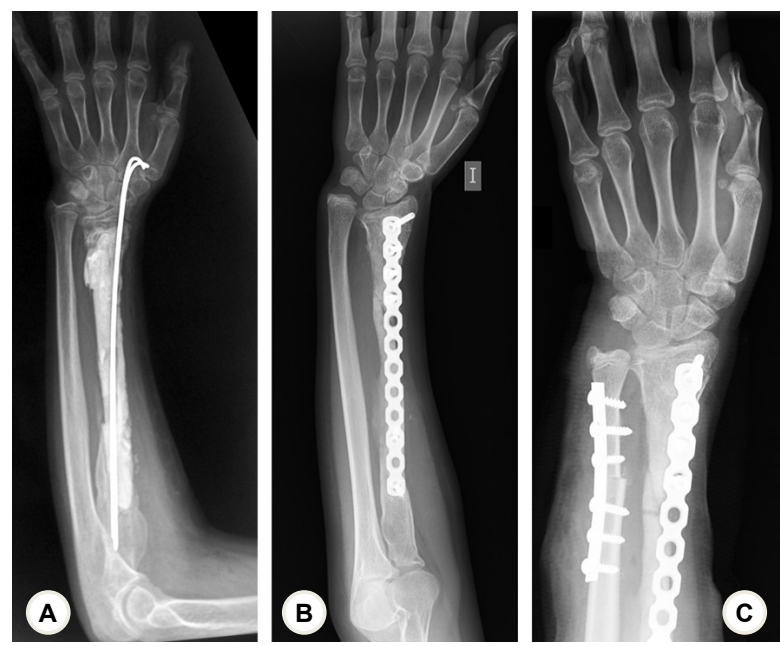

Figura 2. Caso 2. Mujer de 13 años de edad con un defecto óseo de $125 \mathrm{~mm}$ en el radio izquierdo (etiología: osteomielitis crónica por $S$. aureus). A: Radiografía del antebrazo de la primera etapa muestra el espaciador de PMMA impregnado con vancomicina colocado en el defecto óseo y fijado temporalmente con dos agujas Kirschner. B: Radiografía nueve meses de realizada la segunda etapa. El espaciador fue reemplazado por injerto óseo heterólogo y el radio estabilizado con una placa de reconstrucción de titanio. Se evidencia consolidación completa del radio con $12 \mathrm{~mm}$ de acortamiento que ocasiona desviación radial de la muñeca. C: Radiografía de la muñeca luego de osteotomía de acortamiento del cubito.
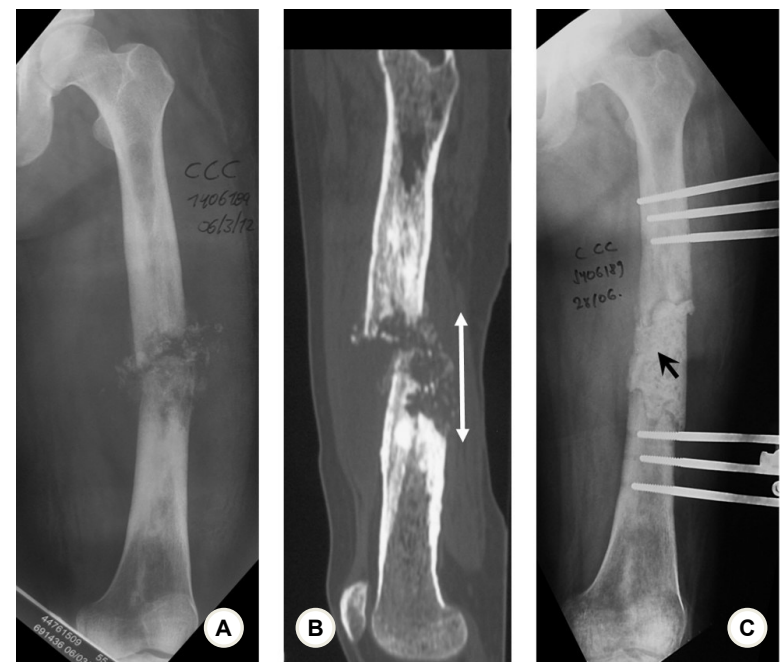

Figura 3. Caso 5. Varón de 26 años con fractura patológica del fémur izquierdo por osteomielitis crónica (cultivo positivo a $S$. aureus). A: Radiografía anteroposterior de fémur izquierdo muestra osteolisis extensa y fractura a nivel del tercio medio de la diáfisis. B: Tomografía del fémur izquierdo evidencia, en su corte sagital, un defecto óseo de hasta $80 \mathrm{~mm}$ (flecha blanca). C: Radiografía de la primera etapa de la técnica de inducción de membrana muestra el espaciador de PMMA impregnado con vancomicina (punta de flecha) ocupando el defecto óseo que ha resultado luego del desbridamiento del tejido óseo infectado.

Es conocido que reconstruir un defecto óseo mediante osteogénesis requiere una curva de aprendizaje larga y uso de fijadores especializados como fijador con anillos circulares de Ilizarov o el fijador hibrido $(29,30)$. Por otra parte, Júpiter y col
(13), detallaron en una serie corta de siete pacientes con defectos femorales tratados con peroné vascularizado, lo complejo de la técnica: realizaron desbridamientos seriados, necesitaron equipos entrenados en microcirugía y tiempos operatorios prolongados, colocaron injertos de peroné a doble columna o en su defecto indicaron tiempo prolongado en descarga hasta lograr la hipertrofia del peroné implantado. La técnica de Masquelet se puede aplicar tanto en miembros superiores como inferiores $(16,18,26,31)$.

En nuestra serie se trató dos DOPT segmentarios a nivel del miembro superior. El cubito (caso 1) consolidó con $2 \mathrm{~mm}$ de acortamiento, el paciente no refirió molestias durante los tres años de seguimiento. Sin embargo, el radio (caso 2) consolidó con un acortamiento de $14 \mathrm{~mm}$, produciéndose una desviación radial de la muñeca y prominencia del cubito distal, se resolvió posteriormente con una osteotomía de acortamiento del cubito (Figura 2). El antebrazo funciona como una doble articulación donde la longitud, alineación axial y rotación correcta de ambos huesos asegura el equilibrio articular en la muñeca y el codo (32). Es recomendable utilizar un fijador externo durante la primera etapa, para asegurar la congruencia radio-cubital tanto distal como proximal.

En DOPT segmentarios en tibia el mayor problema es la cobertura de partes blandas. Si el defecto está
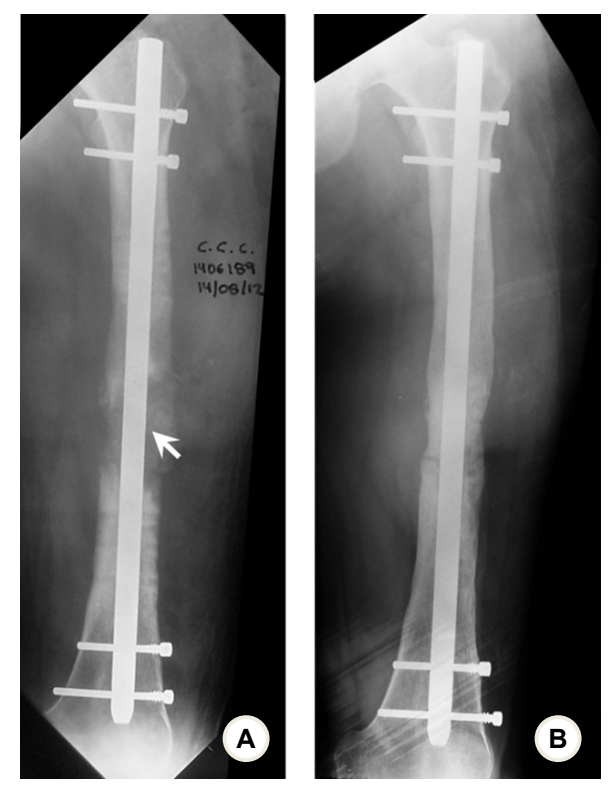

Figura 4. Caso 5. A: Radiografía de fémur de la segunda etapa de inducción de membrana, se ha reemplazado el espaciador de PMMA por injerto óseo esponjoso autólogo (punta de flecha) obtenido de cresta iliaca. B: Radiografía ocho meses de haberse realizado la segunda etapa, se evidencia consolidación del injerto óseo con resolución del defecto femoral. No hay evidencia radiográfica de reactivación de la infección. 
localizado en el tercio proximal o medio de la tibia, un colgajo rotatorio asegura la cobertura del defecto y del espaciador. Si el defecto es extenso y distal, se necesita un colgajo libre con anastomosis microvascular $(3,33)$ En la planificación de la reconstrucción es vital asegurar la oportuna cobertura del hueso y del espaciador, de no ser posible habría que optar por otras técnicas de reconstrucción como el acortamiento primario y alargamiento posterior $(33,34)$.

La masa muscular que rodea la diáfisis femoral asegura una cobertura adecuada del defecto y del espaciador. Sin embargo, las cicatrices de cirugías previas o las múltiples fístulas hacen de la disección a nivel del muslo una tarea muy difícil debido al sangrado excesivo. Una opción al abordaje lateral convencional es el abordaje antero-lateral de Henry, el cual permite abordar la diáfisis femoral separando suavemente el vasto lateral del recto anterior e incidiendo directamente en el músculo crural (35).

El tiempo de permanencia del espaciador en nuestro estudio fue de 8,5 semanas. Aho y col (22), realizaron análisis histoquímico de membranas inducidas y encontraron que la capacidad osteogénica disminuye con el tiempo. La vascularización de las membranas se reduce a menos de $60 \%$ a los tres meses. Mientras que la expresión de factor de crecimiento vascular endotelial (VEFG) es máxima al mes y se reduce a menos del $40 \%$ a los dos meses. No se debe retrasar la segunda etapa a fin de aprovechar al máximo las propiedades biológicas de la membrana inducida (19).

La reconstrucción por etapas asegura un medio aséptico seguro para estabilizar con fijación interna como el enclavado endomedular (Figuras 3 y 4). En nuestra serie el $80 \%$ de los casos fueron estabilizados con fijación interna, sólo en un caso (caso 6) se observó reactivación de una infección por E. coli. Para disminuir el riesgo de recurrencia el desbridamiento debe incluir un margen mayor de $5 \mathrm{~mm}$ de hueso (36).

Una limitación de la técnica es la fuente de injerto esponjoso autólogo. Se puede obtener, entre 20 a $50 \mathrm{cc}$ de la cresta iliaca anterior (37). Es necesario preparar durante el acto quirúrgico ambas crestas iliacas o la cresta iliaca posterior. Un sistema de fresado del canal femoral y aspiración simultanea (Reamer/Irrigator/ Aspirator - RIA) sería una alternativa para obtener volúmenes entre 30 a $90 \mathrm{cc}$ de injerto $(38,39)$. En nuestra serie se utilizó RIA en dos pacientes (caso 9 y caso 19). En un defecto en radio (caso 2) solo se colocó injerto heterólogo, consolidando a los 9 meses.
En casos seleccionados se podría aportar injerto heterólogo únicamente. Los DOPT segmentarios fijados con clavos endomedulares requieren un volumen menor de injerto, comparado con otros sistemas de fijación (19).

El $90 \%$ de los DOPT tratados fueron mayores a $50 \mathrm{~mm}$ y consolidaron el $90 \%$. En un defecto femoral (caso 8) fue necesario agregar un segundo aporte de injerto a los 3 meses de seguimiento, aumentando la tasa de consolidación global hasta $95 \%$. Karger (31), reportó 84 casos (57\% mayores a $50 \mathrm{~mm}$ ) con consolidación de $90 \%$ y $7 \%$ de amputaciones, en nuestra serie no se requirió ninguna amputación.

En conclusión la técnica de inducción de membrana representa una buena alternativa en el manejo de DOPT segmentarios, con tasas adecuadas de consolidación, baja frecuencia de complicaciones, tolerable por el paciente y reproducible en los hospitales de nuestro medio. Las limitaciones de nuestro trabajo son el número relativamente reducido de la muestra, así mismo hubiera sido interesante contar con estudio histopatológico de las membranas inducidas. Se debe estudiar igualmente el alcance de esta técnica en fracturas con compromiso articular.

\section{Declaración de financiamiento y de conflictos de intereses:}

El estudio fue financiado por los autores, quienes declaran no tener conflicto de intereses que resulten del estudio de investigación.

\section{Contribución de autoría:}

JV participó en la recolección y análisis de los datos y en la redacción del artículo; CR, CG y JH participaron en la interpretación de los resultados y en la revisión crítica del artículo.

\section{Correspondencia:}

Julio César Vejarano Solano

Centro de Traumatología y Ortopedia, Hospital Nacional Cayetano Heredia.

Av. Honorio Delgado 262, Lima 31, Perú.

Teléfono: 511942763956

Correo electrónico: julio.vejarano@gmail.com

\section{REFERENCIAS BIBLIOGRÁFICAS}

1. DeCoster T, Gehlert R, Mikola E, Pirela-Cruz M. 
Management of posttraumatic segmental bone defects. J Am Acad Orthop Surg. 2004; 12:28-38.

2. Webb L. Defect nonunion of the lower extremity. Techniques in Orthopaedics. 2001; 16(4): 387-397.

3. Patzakis $\mathrm{M}$, Zalavras $\mathrm{Ch}$. Chronic posttraumatic osteomyelitis and infected nonunion of the tibia: Current management concepts. J Am Acad Orthop Surg. 2005; 13:417-27.

4. Beals R, Bryant R. The treatment of chronic open osteomyelitis of the tibia in adults. Clin Orthop Relat Res. 2005; 433:212-17.

5. Lazzarini L, Mader J, Calhoun J. Osteomyelitis in long bones. J Bone Joint Surg Am. 2004; 86:2305-18.

6. Agner J, Kyle B, Cierny G, Webb L. Diagnosis and management of chronic infection. J Am Acad Orthop Surg. 2011; 19 (S1):S8-S19.

7. Court-Brown Ch. Fracturas de la tibia y del peroné. En: Bucholz R, Heckman J. (Edit). Rockwood \& Green`s Fracturas en el adulto. Madrid: Marban Libros; 2003. p. 1991-92.

8. Pelissier P, Boireau P, Martin D, Baudet J. Bone Reconstruction of the Lower Extremity: Complications and Outcomes. PIast Reconstr Surg. 2003; 111(7): 2223-29.

9. Cierny III G, DiPasquale G. Treatment of chronic infection. J Am Acad Orthop Surg. 2006; 14: S105-S110.

10. Fleming M, Watson T, Gaines R, O'Toole R. Evolution of orthopaedic reconstructive care. Am Acad Orthop Surg. 2012; 20(S1): S74-S79.

11. Pederson W, Person D. Long bone reconstruction with vascularized bone grafts. Orthop Clin N Am. 2007; 38:23-35.

12. Levin S. Vascularized fibula graft for the traumatically induced long-bone defect. J Am Acad Orthop Surg. 2006; 14: S175-S176.

13. Jupiter J, Bour C, May J. The reconstruction of defects in the femoral shaft with vascularized transfer of fibular bone. J Bone Joint Surg Am. 1987; 95: 365374.

14. Wood M. Femoral reconstruction by vascularized bone transfer. Microsurgery. 1990; 11(1):74-9.

15. Mekhail A, Abraham E, Gruber B, Gonzalez M. Bone transport in the management of posttraumatic bone defects in the lower extremity. J Trauma. 2004; 56: 368-378.

16. Masquelet AC, Fitoussi F, Begue T, Muller GP. Reconstruction of the long bones by the induced membrane and spongy autograft. Ann Chir Plast Esthet. 2000; 45(3):346-53.

17. Masquelet A, Begue T. The concept of induced membrane for reconstruction of long bone defects. Orthop Clin Am. 2010; 41: 27-37.

18. Woon CY, Chong KW, Wong MK. Induced membranes -a staged technique of bone-grafting for segmental bone loss: A report of two cases and a literature review. J Bone Joint Surg Am. 2010; 92: 196-201.

19. Taylor B, Frech B, Fowler T, Russell J, Poka A. Induced membrane technique for reconstruction to manage bone loss. J Am Acad Orthop Surg. 2012; 20: 142-150.

20. Giannoudis P, Faour O, Goff T, Kanakaris N, Dimitrou R. Masquelet technique for the treatment of bone defects: tips-tricks and future directions. Injury. 2011; 42: 591-598.

21. Pelissier P, Masquelet A, Bareille R, Mathoulin S, Amedee J. Induced membranes secrete growth factors including vascular and osteoinductive factors and could stimulate bone regeneration. J Orthop Research. 2004; 22:73-79.

22. Aho OM, Lehenkari P, Ristiniemi J, Lehtonen S, Risteli J, Leskelä HV. The mechanism of action of induced membranes in bone repair. J Bone Joint Surg Am. 2013; 95: 597-604.

23. Jaeblon T. Polymethyl methacrylate: Properties and contemporary uses in orthopaedics. J Am Acad Orthop Surg. 2010; 18: 297-305.

24. Webb J, Spencer R. The role of polymethyl methacrylate bone cement in modern orthopaedic surgery. J Bone Joint Surg Br. 2007; 89(7):851-57.

25. Kuehn K, Ege W, Gopp U. Cementos óseos acrílicos: composición y propiedades. Cemento óseo acrílico en el nuevo milenio. Madrid: Ed. Panamericana. 2005. p. 21-34

26. Stafford P, Norris B. Reamer-irrigator-aspirator bone graft and bi Masquelet technique for segmental bone defect nonunions: a review of 25 cases. Injury. 2010; 41(S 2): S72-7.

27. Christian E, Bosse M, Robb G. Reconstruction of large diaphyseal defects, without free fibular transfer, in Grade-IIIB tibial fractures. J Bone Joint Surg Am. 1989; 71:994-1004.

28. DeCoster T, Bozorgnia Sh. Antibiotic beads. J Am Acad Orthop Surg. 2008; 16:674-678.

29. Blum A, Bongiovanni J, Morgan S, Flierl M, Baldy F. Complications associated with distraction osteogenesis for infected nonunion of the femoral shaft in the presence of a bone defect. J Bone Joint Surg. 2010; 92:565-70.

30. Ali F, Saleh M. Treatment of distal femoral nonunions by external fixation with simultaneous length and alignment correction. Injury. 2002; 33:127-34.

31. Karger C, Kishi T, Schneider L, Fitoussi F, Masquelet A. Treatment of posttraumatic bone defects by the induced membrane technique. Orthop Traumatol Surg Res. 2012; 98(1):97-102

32. Jayakumar P. Jupiter J. Reconstruction of malunited diaphyseal fractures of the forearm. Hand (NY). 2014; 9(3): 265-73.

33. Masquelet A, Haas W. Soft-tissue: principles of management. En: Rüedi T, Buckley R, Moran C. (Edit). AO principles of fracture management. Davos: 
AO Publishing; 2007. p. 370-83.

34. El-Rosasy M. Acute shortening and re-lengthening in the management of bone and soft-tissue loss in complicated fractures of the tibia. J Bone Joint Surg Br. 2007; 89:80-8.

35. Bauer R, Kerschbaumer D, Poisel S. Cirugía ortopédica: cadera y femur. Madrid: Marban-Libros; 2004.p.31-33.

36. Simpson A, Deakin M, Jathan J. The effect of the extent of surgical resection on infection-free survival. J Bone Joint Surg. 2001; 83: 403-7.

37. Myeroff C, Archdeacon M. Autogenous bone graft: donor sites and techniques. J Bone Joint Surg Am. 2011; 93: 2227-36.
38. Sagi H, Young M, Gerstenfeld L, Einhorn T, Tornetta P. Qualitative and quantitative differences between bone graft obtained from the medullary canal (with a Reamer/Irrigator/Aspirator) and the iliac crest of the same patient. J Bone Joint Surg Am. 2012; 94 (23):2128-35.

39. Masquelet A, Benko P, Matheron H, Hannouche D, Obert L. Harvest of cortico-cancellous intramedullary femoral bone graft using the Reamer- IrrigatorAspirator (RIA). Orthop Traumatol Surg Res. 2012(2): 227-32.

Recibido: 05/12/2014 Aceptado: 29/03/2014 